\title{
III. Die höfische Gabe als Kommunikationsmedium
}

Die Soziologie verwendet, ausgehend von sozialanthropologischen Erkenntnissen ${ }^{1}$, für die Gesetzmäßigkeit des dem Gabentausch immanenten Prinzips von Gabe und Gegengabe den von Richard Thurnwald eingebrachten und insbesondere von Marshall Sahlins ausformulierten Begriff der Reziprozität ${ }^{2}$ in Abgrenzung dieses Tauschprinzips von einem anderen, nämlich dem ökonomischen Warentausch ${ }^{3}$. Nach Sahlins kann "generalisierte«, »ausgeglichene« und »negative« Reziprozität unterschieden werden. Die generalisierte Reziprozität als bindungsstärkste Form beinhaltet das "trennende Zeitintervall« zwischen Gabe und Gegengabe und ist nicht bestimmt durch deren exakte Entsprechung, weder nach Art, noch nach Umfang ${ }^{4}$. Dies ist hingegen bei der ausgeglichenen Reziprozität der Fall ${ }^{5}$. Negative Reziprozität löst begrifflich das Problem ausbleibender Gegenseitigkeit ${ }^{6}$.

Berking weist freilich richtig darauf hin, daß Reziprozität und Tausch nicht identisch seien 7 . Das Geben der Gabe ziele primär auf Reziprozität und nicht auf Tausch, auf soziale Beziehung und nicht auf Gütererwerb: »Reziprozität stiftet [...] ein positives Verhältnis, Tausch läßt die Partner aneinander uninteressiert. $\aleph^{8}$ Reziprozität ist also nicht Tausch an sich, sondern sozialer Tausch ${ }^{9}$. Als sozialer Tausch kann Reziprozität ${ }^{10}$ auf der makrosoziologischen

1 Siehe oben S. 10, Anm. 7 (Literatur) und oben S. 11, Anm. 9 (zu Marcel MaUSS).

2 THURNWALD 1932, S. 106f:: "The idea is that the person receiving his or her share will be ready tomorrow to give the same to the distributor of today. Today's giving will be recompensed by tomorrows taking. This is the outcome of the principle of reciprocity [...] «. Siehe zu der hier explizit mitnotierten Zeitverschiebung unten S. 125. - Zusammenstellung von Literatur zum Prinzip der Reziprozität unten Anm. 10.

3 Siehe SAHLINS 1965, hier die Darstellung der Unterscheidung der drei Subtypen von Reziprozität, vgl. ARGYLE 1972, S. 169-174.

4 Siehe SAHLINS 1965, S. 147; hier könnte das »Gebet als Gabe«, siehe OEXLE 1976, hier v.a. S. 87ff., eingeordnet werden.

5 Siehe SAHLINS 1965, S. $147 \mathrm{f}$.

6 Siehe ebd., S. 148f.; vgl. GOULDNER, Etwas gegen nichts, 1984.

7 Siehe BERKING 1996, S. 72.

8 HAFERLAND 1989, S. 42.

9 Zu diesem Unterschied v.a. STENTZLER 1975. Vgl. HAFERLAND 1989, S. 4lf., 307f., Anm. 86.

10 Siehe BLAU, Peter M.: Art. »Interaction (IV): Social Exchange«, in: International Encyclopedia of the Social Sciences, Bd. 7, 1968, S. 452-458 und ELWERT, Georg: Art. "Tausch«, in: Wörterbuch der Soziologie, hg. von ENDRUWEIT, TROMMSDORF, Bd. 3, 1989, S. 721-724, dann THURNWALD 1932 (siehe oben Anm. 2) und THURNWALD 1957, hier S. 82-103 die Darstellung von Gegenseitigkeit-Reziprozität; LÉVI-STRAUSS 1967, hier v.a. "soziale Schenkungen « S. 364-366 und "Austauschsysteme (Kommunikations-)« S. 321326; OPPITZ 1975, hier S. 99-104 zur Reziprozität, BLAU 1992, hier Kap. 4: »Social Exchange «, S. 88-114; dazu CLAUSEN 1991, S. 30-35 und ROST 1994, S. 124-132; 
Ebene wiederum als Bestandteil der Theorie sozialer Systeme nach Niklas Luhmann behandelt werden, wonach Reziprozität als »Sonderfall von Konditionierung « definiert ist und dadurch das soziale System des Schenkens als Äquivalent des sozialen Tausches ausweist, diesen quasi durch die Hintertür wieder einführt, obwohl der soziale Tausch selbst systemtheoretisch nicht verankert ist: "Die Leistung des einen wird unter der Bedingung der Gegenseitigkeit von der Leistung des anderen abhängig gemacht - also doppelte Kontingenz reduziert auf doppelte Konditionierung « ${ }^{11}$, die damit den »Nullpunkt aller Kommunikation « aufhebt ${ }^{12}$.

Belegen läßt sich Reziprozität in ihrer materiellen Ausprägung anhand der Quellen am Beispiel des Gabentausches zum neuen Jahr nur in Form des direkten, zeitgleichen Gabentausches, sonst nicht, denn: wo ist der definitive Anfang des Geschenkverkehrs, wer agiert, wer reagiert? ${ }^{13}$ Doch auch wenn in dem im Anhang der vorliegenden Arbeit gegebenen Katalog 14 die im selben

HAFERLAND 1989, S. 307, Anm. 80 (hebt hervor, daß BLAU v.a. auf den verpflichtenden Charakter von Reziprozität abzielt, aber, siehe ebd., Anm. 86, den Unterschied von Reziprozität und Tausch nicht angemessen realisiere); SAHLINS 1965 (siehe oben S. 123, Anm. 3); LÉVI-STRAUSS 1981, hier v.a. Kap. V: „Das Prinzip der Gegenseitigkeit«, S. $106 f f$. (im wesentlich auf MAUSS 1990 aufbauend, vgl. ROST, 1994, S. 45-49); POLANYI 1968 (hier die Darstellung der drei Arten der Aufteilung bzw. der Bewegung von Gütern und Dienstleistungen innerhalb einer Gesellschaft im Überblick: Reziprozität, Redistribution, Marktaustausch); ARGYLE 1972, hier insbes. S. 169-174 zur Reziprozität und den drei grundlegenden Reziprozitätstypen nach SAHLINS 1965; BLAU 1969; LÉVI-STRAUSS 1969; MALINOWSKI, Principle of Give and Take, 1969; MALINOWSKI, Reciprocity, 1969; WILMSEN 1972; FLANNERY 1972; OTTERBEIN 1972, hier S. 28 eine Def. von Reziprozität; EKEH 1974 (freundlicher Hinweis von Klaus R. Schroeter, Kiel, vgl. SCHROETER 1994, S. 102-104 mit Abb. 105 auf S. 103a); STENTZLER 1975; CLAUSEN 1978 (vgl. SCHROETER 1994, S. 104-107, und ROST 1994, S. 157-168); VIVELO 1981, hier v.a. Kap. 10 »Wirtschaftliche Organisation «, dort Grundlegendes zur Reziprozität; GERGEN, GREENBERG, WILliS 1980; PrYOR, GRABURN 1980; WeINER 1980; GoULDNER, Reziprozität und Autonomie, 1984 (in seinen systemtheoretischen Ausführungen zur Reziprozität der strukturellfunktionalen Theorie nach PARSONS 1951 verpflichtet, vgl. SCHROETER 1994, S. 99-102); GOULDNER, Die Norm der Reziprozität, 1984 (hierzu die knappe Bemerkung bei HaFERLAND 1989, S. 307, Anm. 79); GOULDNER, Etwas gegen nichts, 1984; Social exchange Theory, hg. von COOK, 1987, und SCHROETER 1994, S. 90-114. - Eine Einzelstudie bietet JOHNSON 1974.

11 LUHMANN 1991, S. 186, vgl. LUHMANN, Gesellschaft der Gesellschaft, Teilbd. 2, 1997, S. 651 (LUHMANN konzentriert seine Ausführungen hier allerdings auf die regulative Funktion der Reziprozität in segmentären Gesellschaften und sieht im Vergleich dazu die Reziprozitätsverhältnisse in stratifizierten Gesellschaften deformiert, siehe ebd., S. 659).

12 Vgl. HAFERLAND 1989, S. 37, S. 38: »Reziprozität ist zunächst nichts anderes als die Realisierung oder Wahrnehmung eines Maßes, das dem Einander eine für beide Seiten voraussehbare Form gibt.«

13 Vgl. den diesen Umstand treffend bezeichnenden Untertitel zum Kap. "Anlässe« bei BERKING 1996, S. 30: „Ein Geschenk ist ein Geschenk, ist ein Geschenk [...] « Vgl. LuHMANN, Gesellschaft der Gesellschaft, Teilbd. 2, 1997, S. 651f.

14 Siehe Anhang - Katalog/Jahreslisten. 
Jahr jeweils gleichzeitig als Schenker und Beschenkte auftretenden Personen kursiv gesetzt sind, scheint dies nur auf den ersten Blick die ideale, weil zeitgleiche Form der Reziprozität zu sein. Denkbar wäre auch, daß hier Reaktionen auf Vorjahresgeschenke oder Antizipationen auf die Geschenke des nächsten Jahres vorliegen und nur ein »zufällig« zeitgleiches Zusammentreffen von Gabe und Gegengabe stattfindet ${ }^{15}$. Immerhin gilt: »Wer sofort erwidert, sich unmittelbar entlastet, negiert die der Interaktionsform des Schenkens eigenen Intention, sich verpflichten zu lassen oder sich verpflichtet zu fühlen. «16 Pierre Bourdieu hat erkannt, daß es »das Gabe und Gegengabe trennende Zeitintervall [ist], das eine Tauschstruktur als irreversibel wahrzunehmen gestattet, die stets bedroht ist, als reversibel, d.h. anderen und sich zugleich als obligatorisch und interessenbestimmend zu erscheinen. ${ }^{17} \mathrm{Da} ß$ »das Zeitgeschenk oder die Vergeudung von Zeit [...] eine der kostbarsten Gaben [bildet] « 18 kann hier nur angedeutet werden ${ }^{19}$. Gleichwohl ist eine (wie auch immer geartete) Gegenseitigkeit elementarer Bestandteil des Gabentausches, vor allem in zeitlicher Perspektive, so daß statt Reziprozität besser von $»$ Reziprozitätszyklen« zu sprechen ist ${ }^{20}$. Dieser Begriff befreit das system-

15 Siehe EWERT, HIRSCHBIEGEL 2000, eine diesem Umstand gewidmete statistisch-analytische Untersuchung am Beispiel des burgundischen Geschenkverkehrs zum neuen Jahr Die Parallele zum Weihnachtsfest ist unübersehbar (siehe auch ISAMBERT 1976). Für die weihnachtliche Bescherung gelte, siehe BERKING 1996, S. 34-42, daß es hier keine über das Fest hinausreichenden Schulden gebe wegen der Gleichzeitigkeit von Gabe und Gegengabe. Es ist aber zu unterstellen, daß frühere Erfahrungen bei diesem Fest wie auch beim vorliegenden Fall der Neujahrs»bescherung « eine erhebliche Rolle als Motivation des Schenkens spielen müssen und nicht nur die müberraschende Konformität unbewußter Regelbefolgung" wie ebd., S. 41, behauptet wird. Siehe die Ausführungen unten S. 274ff.

16 BERKING 1996, S. 22, entspr. BourdieU 1979, S.219-221, hier S. 220. Vgl. ClaUSEN 1991, S. 67: „Für den Empfänger eines Gegengeschenks kann es sogar verletzend sein, wenn nur mit `Gleichwertigem` erwidert, also quasi bezahlt wird. Denn dieser Sachverhalt weist darauf hin, daß der Interaktionspartner quitt sein will und kein weiteres Interesse an einem Austausch hat. Zum reziproken Tausch gehört nicht, daß der Wert der Güter mit mathematischer Genauigkeit gemessen wird.«

17 Siehe BourDiEU 1979, S. 335f. und BouRdiEU 1996, vgl. CLAUSEN 1991, S. 61f. und LUHMANN, Gesellschaft der Gesellschaft, Teilbd. 2, 1997, S. 652f.

18 BourdiEU 1979, S. 350. Siehe auch VEBLEN 1987, S. 51ff. Vgl. DERRIDA 1993.

19 Siehe zur Gastfreundlichkeit oben S. 55, Anm. 89.

20 Vgl. GOULDNER, Die Norm der Reziprozität, 1984, S. 79f. SCHROETER 1994, S. 99-102 spricht in Anlehnung an GoULDNER, Reziprozität und Autonomie, 1984, regelrecht von einem »Reziprozitätssystem «. Reziprozitätszyklen sind nach BERKING 1996, S. 76 kein Nullsummenspiel, sondern der modus operandi sozialer Differenzierung, vgl. HAFERLAND 1989, S. 38. Ebd., S. 40 wird das entscheidende Ergebnis zyklisch ablaufender Reziprozität mit den Worten zusammengefaßt: »Da sie sich zyklisch organisiert, hinterläßt sie das Gefühl gegenseitiger Verpflichtung, wenn auch noch weniger das Gefühl gegenseitiger Rechte.« Schiere Gewohnheit oder bloßer Anlaß scheinen damit als treibende Kräfte des Neujahrsgeschenkverkehrs auszuscheiden. Zudem müssen die dokumentierten Gaben nicht zwangsläufig in Gaben ihre Entsprechung finden und Gabe und Gegengabe nicht identisch sein. CHEAL 1986 hat eine statistische Erhebung reziprozitärer Beziehungen am Beispiel des 
theoretisch festgelegte Schenksystem auch von seiner vordergründigen Statik, und Reziprozität hat als der reduktiv-kommunikative Selektionsprozeß in diesem Sinn zentrale Bedeutung nicht nur für die Bestandserhaltung des Systems, wodurch Reziprozität ausgewiesen ist als Funktion des Schenksystems. Somit erscheint Reziprozität auf der sozialwissenschaftlich-theoretischen Ebene einerseits als konstitutiv für dieses System, ist damit andererseits aber auch strukturierendes Prinzip für die innersystemische Kommunikation und kann hierdurch letzten Endes als das Kommunikationsmedium aller Systeme des sozialen Tausches charakterisiert werden ${ }^{21}$.

Als Transportmittel reziproker Kommunikation hat die Gabe zu gelten, damit ist sie auch »die Ressource, an der Reziprozität immer wieder demonstriert [werden kann]. (22 $^{22}$ Die Gabe übernimmt Kommunikations- und Steuerfunktionen für das Schenksystem und hat als Träger von Reziprozität die Aufgabe, die symbolisch generalisierte Übertragung von Selektionsleistungen sicherzustellen, "ohne daß die in ihrer Verwendung implizierten Vorverständnisse jeweils neu behandelt oder beschlossen werden müssen. « $^{23}$ Eine in diesem Sinn definierte Gabe als Kommunikationsmedium in praktischer Entsprechung des theoretischen Reziprozitätsprinzips eines höfischen Teilsystems könnte als zuallererst höfische Gabe interpretiert werden und ließe sich somit über theoretische Bezüge hinaus ihrer historischen Dimension zuweisen: »Die wirklich höfische Gabe muß Ehrengabe sein, denn sie muß freiwillig gegeben werden. Weder darf sie von der Erwartung einer Gegengabe oder verpflichteter Dankbarkeit geleitet sein, noch darf sie aus bloßer Verpflichtung für ein vorausgehendes Verhalten gegeben werden. So jedenfalls sähe sie im Idealfall aus.«24 Selbstverständlich ist, wie zu zeigen sein

Weihnachtsfestes 1983 unternommen und festgestellt, daß in fast $50 \%$ aller Fälle der Empfänger des wertvollsten Geschenks zugleich der Schenker des wertvollsten Geschenks ist. Bei den restlichen $50 \%$ herrscht also wahrscheinlich keine in diesem Sinn ausgeglichene Reziprozitätsbalance: »Distributive Gerechtigkeit zielt nicht auf ausgeglichene Bilanzen, sondern auf die temporäre Balance zwischen den Graden der Ver- und Entschuldung", BERKING 1996, S. 24.

21 Vgl. VOWINCKEL 1995, S. $116 \mathrm{f}$.

22 HAFERLAND 1989, S. 41.

23 WrLLKE 1991, S. 161.

24 BERKING 1996, S. 191 nach HAFERLAND 1989, S. 151f. - Einschränkend gilt, daß die häufige Wiederholung von Formeln wie donnez en recompensacion des bons et agreables services qu'il lui a faiz ou temps passé, hier am Beispiel des Neujahrsgeschenks für Pierre Gorremont 1419, ACO B 1601, fol. 62 (und verwendet auch bei anderen Gelegenheiten, so aus Anlaß eines Hochzeitsgeschenks für denselben, ACO B 1601, fol. 62: pour consideracion des bons et agreables services qu'il a faiz ou temps passé, fait chascun jour et espere que encores face ou temps avenir), nicht zu verwechseln sind mit dem Ausdruck von Dankbarkeit. Dankbarkeit kann nicht als eines der leitenden Motive des höfischen Schenkens eingeführt werden, weil Dank in Form von Dankbarkeit ja die sin der Interaktionsform des Schenkens institutionalisierte Gefühlsnorm« sei (man dankt also nicht, sondern erwidert), siehe BERKING 1996, S. 46, und zudem habe sich, so ebd., S. 51, der vorneuzeitliche Gaben- 
wird, dies tatsächlich der Idealfall, denn nicht jede Gabe am Hof ist auch höfische Gabe. Die höfische Gabe allerdings kann als Gabe bestimmt werden, die dem (materiellen) Prinzip der Gegenseitigkeit nicht unterworfen scheint, und tatsächlich zerreiße sie nach Helmuth Berking »das Kontinuum des Gebens und Nehmens, der Schuld und der Schuldenbegleichung und setzt sich autonom als einen allein durch die Dialektik der Ehre bestimmten, einmaligen Akt.«25 In diesem Zusammenhang erscheint Freigebigkeit, die vornehmste der ritterlich-höfischen Tugenden ${ }^{26}$ - deren einziges Mittel eben

tausch noch in den »Fallstricken von Status und Prestige« befunden und konnte nicht »unabhängig von sozialen Positionszuschreibungen gestaltet« werden: "Dankbarkeit ist ein modernes Gefühl«, ebd., S. 52. So gesehen wäre Dankbarkeit in höfischen Gesellschaften unzulässiges Surrogat für Anerkennungszuweisungen im Rahmen der dort herrschenden reziprozitären Beziehungen.

25 BERKING 1996, S. 191 (kursive Schreibweise im Original). Anerkennung ist somit die Ressource, damit die Gabe als Ressource von Reziprozität dienen kann, vgl. HAFERLAND 1989, S. 43.

26 Dels $V$. bons aibs per c'oms es puls honratz - Es larguezal premiers, qui ver en tria, E-l segons es pres de cavalaria - Dels $V$. bons aibs per c'oms es puls honratz - Es larguezal premiers, qui ver en tria, - E.l segons es pres de cavalaria - Car per aquel es totz hom plus duptatz - Car per aquel es totz hom plus duptatz nach KÖHLER 1962, S. 250, Anm. $89 \mathrm{zu}$ seinem Beitrag "Reichtum und Freigebigkeit in der Trobadordichtung", ebd., S. 45-72, siehe hier v.a. S. 66f. - Als "Queen of Mediaeval virtues« (so der Titel des Aufsatzes WHITNEY 1923) ist liberalitas, largitas, largesse Zentralbegriff adliger Mentalität im späten Mittelalter (der freilich nicht erst dann seine Bedeutung fand, vgl. etwa zur frühmittelalterlichen "Geisteshaltung" DUBY 1977, S. 52-60, zur Verschwendung ebd., S. 237ff.) »in jeder Formu (PARAVICINI 1994, S. 6). - Zu den historisch faßbaren Wurzeln von Freigebigkeit BRUCK, Eberhard Friedrich: Paulus, die Kirchenväter und der »Fröhliche Geber" im Römischen Recht: Liberalitas und Animus donandi, in: BRUCK, Über römisches Recht im Rahmen der Kulturgeschichte, Berlin, Göttingen, Heidelberg 1954, S. 101-136, siehe hier v.a. "Aristoteles und die Kunst des IRichtigen Gebens(", S. 106-108. BRUCK weist ebd., S. 125 mit weiteren Nachweisen auch auf den (römischen) Unterschied zwischen liberalitas und largitas hin: liberalitas sei die selbstlose Freigebigkeit im Privatleben gewesen, largitas habe der Kennzeichnung eigennütziger öffentlicher Gaben für politische Zwecke gegolten. Die philosophische Sichtweise bei REINER, Hans: Art. "Freigebigkeit«, in: Historisches Wörterbuch der Philosophie, hg. von RITTER, BILER, Bd. 2, 1972, Sp. 1063f. - Zum gegenläufigen Muster, der Verschwendung (zur folle largesse siehe GUERY 1984, S. 1248, Literaturhinweise ebd., S. 1266, Anm. 47), die neben dem Geiz als Gegensatz zur Freigebigkeit zu gelten hat, grundsätzlich BERGFLETH 1975 (eine eher philosophisch ausgerichtete "Theorie der Verschwendung" auf der Grundlage kritischer Überlegungen zu Marcel MAUSS in Anlehnung an Georges BATAILlEs "Begriff der Verausgabung«: BATAILLE 1975. Vgl. die Ausführungen BERKING 1996, S. 85-91), vgl. KRABS 1996, hier zur Verschwendung und zur Philosophie des höfischen Schenkens S. 99ff. bzw. S. 109ff., siehe exemplarisch (Karl VI.) oben S. 76f. und oben S. 77, Anm. 21 sowie oben S. 86, Anm. 72. SOMBART 1922 erklärt die „Entstehung der modernen Welt aus dem Geist der Verschwendung", siehe hier v.a. S. 83-103. - Konkret auf Zeitraum und Problematik bezogen, den Gegensatz zwischen largesse und charite thematisierend (in der Hauptsache nach Christine de Pisan, Le Livre de la Paix, ed. WILLARD, 1958) GUERY 1984, hier v.a. S. 1245 1254: "La controverse sur les dons: largesse du prince et misère du peuple«, vgl. BELL 1962. - Durchgängiges Muster der Fürstenspiegel ist der Appell an die ritterlich-höfischen 
das Geben ist ${ }^{27}$ - als ursächlich verantwortlich für die Herstellung von Ehre28. Im Rosenroman des Guillaume de Lorris wird Freigebigkeit allegorisch beschrieben: "Freigebigkeit trug ein ganz neues Kleid aus orientalischem Purpur. Ihr Gesicht war schön und wohlgeformt; aber ihr Hals war entblößt, denn sie hatte soeben einer Dame vor einem Augenblick ihre Brosche geschenkt; aber es stand ihr nicht schlecht, daß ihr Ausschnitt offen und ihr Hals entblößt war, so daß ihre zarte Haut durch das Hemd seine weiße Farbe zeigte.« Diese Verse stehen am Ende einer genauen Beschreibung von Freigebigkeit: "Sie war aus dem Geschlechte Alexanders und freute sich über nichts so sehr, wie wenn sie sagen konnte: ^Nimm<. Selbst der elende Geiz war nicht so sehr aufs Nehmen bedacht wie Freigebigkeit aufs Geben; und Gott ließ ihr all ihre Güter sich vermehren, so daß sie niemals so viel geben konnte, wie sie im Überfluß hatte. Sehr gepriesen und gelobt wurde Freige-

Tugenden. Es war die schwierige Gratwanderung zwischen Geiz und Verschwendung, die fürstlichem Verhalten abgefordert wurde. So mahnt Jean Gerson, Theologe und Kanzler der Pariser Universität, den König und die Fürsten, nicht zu freigebig zu sein, weil dies das Schmeichlertum fördere und das Volk zu sehr belaste, siehe RöDER 1933 (vgl. KRYNEN 1981, pass.), S. 69 (zur Literaturgattung HADOT, Pierre: Art. „Fürstenspiegel«, in: RAC, Bd. 7, 1972, Sp. 555-632). Honoré Bonet wiederum mahnt in seinem »L'arbre des batailles «: "Next a king, and every prince, should be temperate in all his works and in all his deeds, and should be bounteous and charitable and pitiful to the poor, and should love and comfort them with measure and wisdom, for if a lord puts no measure to his largesse he cannot be thought wise«: Honoré Bonet, L'arbre des batailles, ed. CoOPLAND, 1949, S. 212. Eine Forderung, die sich erst längerfristig durchgesetzt hat (vgl. etwa Machiavellis Ratschläge zur Sparsamkeit: Den Fürsten sollte nicht kümmern, wenn er in den Ruf kommt, knauserig zu sein, siehe nach der Übers. Nicolò Machiavelli, Il Principe, hg. von RIPPEL, 1986, S. 123-127), denn zunächst ist Verschwendung demonstrativer Konsum, siehe unten S. 131 mit den entspr. Anm.

27 Vgl. BERKING 1996, S. 192. Freigebigkeit wird bei HAFERLAND 1989, S. 151 ff. mit weiteren Nachweisen am Beispiel des Zehnten Buches von Thomasins "Wälschem Gast« interpretiert (nach der Ausgabe Thomasin von Zirclaria, Der Wälsche Gast, hg. von RÜCKERT, 1965).

28 Zum mittelalterlichen Ehrbegriff zunächst ECKERMANN, Karl Willigis: Art. "Ehre«, in: LexMA, Bd. 3, 1985, Sp.1662f. mit weiteren Nachweisen, ENGELS, Odilo: Art. »Honor imperii«, in: ebd., Bd. 5, 1990, Sp.119 und ZUNKEL, Friedrich: Art. »Ehre, Reputation«, in: Geschichtliche Grundbegriffe, hg. von BRUNNER, CONZE, KOSELLECK, Bd. 2, 1975, S. 1-63 mit weiteren Nachweisen, hier S. 6ff., 17ff., EHRISMANN 1919 zur Ehre als eine der »Grundlagen des ritterlichen Tugendsystems « nach literarischen Zeugnissen aus dem deutschsprachigen Raum (dazu kritisch NEUMANN 1951; ebenfalls KORTÜM 1996, S. 55, der die Diskrepanz zwischen Ideal und Wirklichkeit nicht angemessen berücksichtigt sieht - vgl. WINTER 1965, S. 78f. zur Freigebigkeit mit einschlägigen Hinweisen), neueren Datums EHRISMANN 1995. Eine soziologische Betrachtung gibt GUTTANDIN, Soziologie der Ehre, 2 Bde., 1989 (vgl. GUTTANDIN 1993), siehe hier v.a. den Beitrag ZINGERLE 1989 (zur lexikal. Kurzinformation: ZINGERLE, Arnold: Art. "Ehre«, in: Wörterbuch der Soziologie, hg. von ENDRUWEIT, TROMMSDORF, Bd. 1, 1989, S. 132f.). "Höfische Ehre« behandelt ELIAS 1983, S. 145ff. Philosophischer Natur ist REINER 1956 (siehe REINER, Hans: Art. "Ehre«, in: Historisches Wörterbuch der Philosophie, hg. von RITTER, BILER, Bd. 2, 1972, S. 319-328, hier v.a. S. 320f.). 
bigkeit; die Weisen sowohl wie die Toten hielt sie fest in ihrer Gewalt, so viel hatte sie mit ihren guten Gaben erreicht. Selbst wenn jemand sie hassen würde, so glaube ich, daß sie ihn durch ihre großen Dienste zu ihrem Freund machen würde; und deshalb besaß sie nach ihrem Wunsch die Liebe der Armen und der Reichen. Sehr töricht ist ein hoher Herr, der knauserig ist. Ein hoher Herr kann kein Laster haben, das ihm so sehr schadet wie der Geiz; denn ein Geiziger kann keine Herrschaft erwerben noch weites Land, denn er hat nicht genügend Freunde, die ihm zu Willen sind. Wer aber Freunde haben will, dem sei sein Gut nicht zu teuer; vielmehr erwerbe er sich Freunde durch schöne Geschenke; denn ganz in der Weise, wie der Magnetstein das Eisen geschickt an sich zieht, so zieht das Gold, das man verschenkt, und das Silber die Herzen der Leute an. $\otimes^{29}$

Als Ziel der Gaben wird hier genannt: Freundschaft, Freunde, »die Herzen der Leute « - mit keinem Wort die konkrete Gegengabe, vielmehr erscheinen hier die Gaben als »marques d'une amitié réciproque «30. Also doch auf Gegenseitigkeit gründende Beziehungen? Diese Frage muß selbstverständlich bejaht werden, aber die richtige höfische Antwort auf die höfische Gabe liegt nicht in der Gegengabe, sondern eben in der Ehrzuweisung: lez Roys et lez Impereurs sont donataires, par consequant ilz sont seigneurs, steht im "Songe du Vergier ${ }^{31}$ geschrieben. Hier klingt auch der begrenzte Personenkreis an, der dieser Freigebigkeit in aktiver wie passiver Weise fähig ist, nämlich im allgemeinen Fall der Adel ${ }^{32}$, im theoretischen Sinn die "Gleichgestellten«, in stilisierter Form die »Höflinge «33: »Ehre und Anerkennung sind hier im Idealfall die einzig bedeutsamen Ressourcen, die mittels der Gaben und motiviert durch ein freigebiges, der reinen Absicht des Gebens eingedenkendes Handeln verteilt werden. $^{34}$ Reziprozität gelingt, weil »Freigebigkeit in der höfischen

Beide Zitate nach der Ed. OTT, Der Rosenroman, Bd. 1, 1976, S. 132, 134, Vers $1164-$ 1172 , dt. Übers. ebd., S. 133, 135 (siehe auch oben S. 97, mit Anm. 122). Vgl. STAROBINSKI 1994, S. 31, 183.

30 VIGIER 1674, S. 5 nennt so die étrennes.

31 Le Songe du Vergier, ed. SCHNERB-LIÈVRE, 2 Bde., 1982, hier Bd. 1, S. 123 (Buch II, Kap. CXLV).

32 Vgl. BERKING 1996, S. 192 mit dem Hinweis, daß den Armen und Bedürftigen die Ehre ja wenig nutze, ihnen gewähre man das Almosen. Dies ist m.E. aber nur die eine Richtung und vernachlässigt zudem die »unbeteiligten Dritten«. Freigebigkeit ist Armen natürlich nicht möglich, aber in Treue sich auszahlende Anhänglichkeit. Außerdem kann Ehrzuweisung indirekt erfolgen, indem der Akt der Almosenvergabe von den Standesgenossen positiv bewertet wird.

33 Eine Figur, die sich dann v.a. ab der Renaissance entwickelt, vgl. ANGLO 1978 (mit Nennung der wichtigsten Höflingsliteratur), S. 52 nach Lorenzo Duccis »Arte Aulica» von 1601, hier allerdings zynisch gemeint: "Der knickrige, knausrige und absolut nicht großzügige Fürst ist ein wertloses Lebewesen, denn er ist ohne Nutzen für die menschliche Gesellschaft."

34 BERKING 1996, S. 192. 
Interaktion reziprok typisiert wird und in bezug auf die Binnenstruktur der Gruppe die Haltung markiert, die für die symmetrische Verteilung von Anerkennung maßgeblich ist. « 35 Das ist neben dem trennenden Zeitintervall von Gabe und Gegengabe (das Reziprozität zur Reziprozitätserwartung werden läßt) das entscheidende Moment, das aus dem Gabentausch am Hof integrativ wirksamen höfischen Geschenkverkehr werden läßt und die für den Erhalt des Systems wichtigen identitätsstiftenden reziproken Bindekräfte entfalten kann 36: "Die Freiwilligkeit der Verpflichtung wird möglich, weil die strikte Zäsur zwischen Gabe und Gegengabe das Verpflichtetsein nicht mehr als eine Antwort auf empfangene Wohltaten, sondern als einen eigenständigen Akt konnotiert, dessen symbolischer Wert dem der Freigebigkeit nun korreliert. Freigebig zu sein, sich verpflichten zu lassen und sich verpflichtet zu zeigen, sind nur mehr zwei Seiten des formvollendeten Ausdrucks der einen höfischen Bindung, deren innere Struktur und ideale Gestalt Reziprozität bildet. (37 $^{37}$

Und, auch hier noch Berking folgend, „der Adel zumindest weiß oder könnte doch wissen, wem er was, wie und warum zu geben hat. « ${ }^{38}$ Und der Adel weiß dies durchaus. Freigebigkeit als höfisches Verhalten ist nicht nur zielorientiert motiviert durch den Erwerb ${ }^{39}$ und die Akkumulation des symbolischen Kapitals der Ehre ${ }^{40}$ - gemessen an den den einzelnen Geschenken eignenden Werten und damit distinktiven Charakters. Das entscheidende Kriterium hierbei ist die Konvertierbarkeit in soziales Kapital in Form der Etablierung von Anerkennungsverhältnissen und deren Reproduktionsfähigkeit, wozu nach Bourdieu weine unaufhörliche Beziehungsarbeit in Form von ständigen Austauschakten erforderlich [ist], durch die sich die gegenseitige Anerkennung immer wieder neu bestätigt. «4l Das bedeutet: »Bei der Beziehungsarbeit wird Zeit und Geld und damit, direkt oder indirekt, auch ökonomisches Kapital verausgabt. Ein solcher Einsatz ist nur rentabel, ja er ist überhaupt nur denkbar, wenn eine besondere Kompetenz - nämlich die Kenntnis genea-

\section{BERKING 1996, S. 192.}

36 HAFERLAND 1989, S. 49 geht soweit, daß er über Reziprozität die Sicherung der kulturellen Identität höfischer Gesellschaften gewahrt sieht. Eine erklärende historische Interpretation dieser Entwicklung im 15. Jh. bei BORNSTEN 1975, S. 16f., hier S. 17 im Versuch, eine Antwort auf die thesenhaft formulierte Frage zu finden, warum die höfischen Feste der Zeit begannen, einen dem potlatch ähnlichen Charakter anzunehmen: „Chivalry moved from the realm of business and work, to the realm of social ritual and ceremony, to the realm of play [...] social rituals and games [...] helped to create a sense of cohesion, seperateness, and superiority among the wealthy class [...] Rituals of chivalry and courtesy served to identify the upper class as a seperate group."

37 BERKING 1996, S. 195.

38 Ebd.

39 Vgl. Elias 1983, S. 157.

40 Vgl. BOURDIEU 1979, S. 335-378 (Kap. 5 »Symbolisches Kapital und Herrschaftsformen«).

41 BOURDIEU 1983, S. 193 (kursive Schreibweise im Original). 
logischer Zusammenhänge und reeller Beziehungen sowie die Kunst, sie zu nutzen - in sie investiert wird. « 42

Denn Freigebigkeit ist auch Ausdruck einer Standesqualität, die, von Norbert Elias als »Prestigeverbrauch» und »Statuskonsum « ${ }^{43}$ bezeichnet (hierin Thorstein Veblens $»$ Theorie der feinen Leute ${ }^{44}$ kritisch folgend), quasi die geadelte Seite der Verschwendung darstellt (freilich wird dieses Verhalten erst im weiteren historischen Verlauf zur vollen Entfaltung kommen). Zuweisung von Ehre erfolgt entsprechend der innerhöfischen Ansiedlung des Schenksystems selbstverständlich nicht »seitens der machtunterworfenen Umwelt«, wie dies beim Hof der Fall ist, sondern eben durch Standesgenossen, die für alter und ego dann natürlich zur Umwelt gehören. Wie homogen oder heterogen die Gruppe der »Standesgenossen « tatsächlich ist, ob diese Gruppe möglicherweise auch aus Personen besteht, die sich nicht dem "Adel« zurechnen lassen oder/und durchwegs identisch ist mit dem am Beispiel des Januarblattes aus dem Stundenbuch des Herzogs von Berry vorgeführten Personenkreis, wird zu zeigen sein.

44 VEBLEN 1987, hier v.a. S. 79ff. Vgl. ebd., S. 85 (lang vor Marcel MAUSS schon das potlatch als Beispiel anführend) den Gedanken stellvertretenden Konsums, wie er durch den Gast erfolgt, 87 zum Zusammenhang von Prestige und Geschenk. 
